\title{
Study of the influence of the pressure and rotational motion of 3D substrates processed by magnetron sputtering: A comparative study between Monte Carlo modelling and experiments
}

\author{
Martin Evrard $^{\mathrm{a}}$, Aurelien Besnard ${ }^{\mathrm{b}}$, Stephane Lucas ${ }^{\mathrm{a}, *}$ \\ ${ }^{a}$ University of Namur, LARN-NISM, 61 rue de Bruxelles, B-5000, Namur, Belgium \\ ${ }^{\mathrm{b}}$ Arts et Metiers ParisTech, LaBoMaP, Rue porte de Paris, F-71250, Cluny, France
}

\section{Keywords:}

Monte-carlo simulation

PVD

thickness uniformity

3D complex substrate

macroscopic shadowing

motion

\begin{abstract}
A B S T R A C T
Over the last ten years, low pressure plasma solutions for materials surface treatment have been remarkable. Nevertheless, the deposition of films with a uniform thickness on 3D complex shapes is still a challenge for various deposition systems. In several cases, concavities and different substrate orientations and motions lead to macroscopic shadowing and affect the thickness uniformity. The objective of this work is to describe a modelling method able to predict the layer thickness on any surface of 3D substrates in motion and subject to vapour transported in a low pressure vessel. The meshing of objects with Delaunay-triangulation enables the modelling of complex shapes. The deposition process consists of several Monte Carlo simulations involving first the computing of the angular and energy particles distribution from the source, second their transport through the chamber and last the deposition on a meshed substrate. The algorithm is optimised with a "cell-list-linked-like" method and differs from existing models by the computation speed.

The benchmarking between simulation and experimental results for $\mathrm{Cr}$, $\mathrm{Ag}$ and $\mathrm{Ta}$ deposition at various pressures and on moving complex substrates with several shadowed faces is presented. Moreover, particle energy distribution will be discussed for each sample surface, mode and pressure.
\end{abstract}

\section{Introduction}

Low pressure plasma-based Physical Vapour Deposition (PVD) is a mature technique used nowadays to deposit a large set of coatings on a large variety of substrates for a wide range of applications. Whatever the application, controlling the film thickness uniformity is very important.

In microelectronic manufacturing, at a micron or sub-micron scale, PVD is used for the deposition of barriers or interconnecting metals. For a good quality barrier, the film thickness must be homogeneous over the entire contact. Non-conformal PVD step coverage may lead to the diffusion of copper in the surrounding materials, which would degrade their properties. Also, PVD is used to fill the trenches and vias between the various layers on an electronic chip, and the filling of trenches with a uniform layer has been the topic of intensive research for several years [1].

In optics, film thickness uniformity is of prime importance for multilayer films of extreme precision: telescope mirrors for gravitational wave detectors [2], optical filters [3], anti-reflective coatings on large substrates [4], and protection filters for lasers [5,6]. Non-uniform films deposited by PVD directly affect the physical properties of the single- or multi-layer structure and thus its performance.

The thickness uniformity issue is also a challenge for coatings deposited on components (e.g. cutting tools) with the purpose of improving their tribological properties: the worst case scenario leads to film cracking or peel off because of layers subjected to a gradient of tensile stress [7].

Several experimental strategies have been implemented to improve thickness uniformity over non-flat substrates. The first is to act on the directionality of the condensing species. This can be achieved experimentally by using collimated sputtering, ionized metal physical vapour deposition, and/or by appropriately setting up experimental process parameters like the substrate holder geometry, the vacuum pressure, etc. Honeycomb shaped stainless steel collimators have been used to achieve conformal thickness on large mirrors and also to smooth the multilayer interfaces [8]. Ionized metal physical vapour deposition is a process in which the sputtered metal atoms from the target are ionized by a secondary plasma or by the primary excitation itself, like in

\footnotetext{
* Corresponding author.

E-mail address: stephane.lucas@unamur.be (S. Lucas).
} 
HIPIMS. Ions are accelerated toward the substrate and hit the substrate with a normal direction due to the influence of a sheath. It limits the shadowing process and improves the substrate coverage. Experimental parameters like pressure also have an influence. Indeed, high pressure plasma discharge involves multiple collisions in the gas phase and reduces the species. It helps cover the local areas of parts which are not in line of sight with respect to the material source.

The second experimental strategy relates to samples in motion. B. Rother developed a simple analytical model to predict the effects of substrate-carrier arrangements (different numbers of satellites, different gear ratios between the satellite and the base, rotation frequencies, etc.) on multilayer deposited on flat substrate with a three-fold rotation system $[14,15]$. This model is used in several works by Panjan et al. to highlight influence of rotation on multilayer stoichiometry and periodicity [16-19]. A similar approach has been also used to investigate the multilayers formation as a function of substrate rotation speed in the case of co-sputtering deposition by confocal sources [20]. Unfortunately, all these work are applicable only for flat substrates. In the field of optics, in addition to the use of simple or planetary rotation stages, precisely shaped masks are designed for the purpose of improving film thickness uniformity but also to obtain specific thickness profiles [9-11]. In the field of tribology, complex 3D parts are loaded in large chambers with multiple rotation substrate holders, which take into account the parts geometry $[12,13]$.

Trial and error is still the most common method to optimise the masks, how the parts have to be positioned in the machine or at which process-point values a deposition has to take place to achieve uniform coverage. Also, local film thickness evaluation at various locations of a complex part is generally not undertaken. Indeed, it is a tedious task requiring destructive analysis. Instead, a geometrically simplified witness sample (i.e. a piece of silicon) is generally used to evaluate the average film thickness, but this process is rarely satisfactory.

Process optimisation would be easier if an accurate simulation tool was available. It would have to be able to predict thickness uniformity of coatings deposited on geometrically complex substrates positioned on a given sample holder of a batch coater, possibly submitted to a shadowing mask. Knowing the impact of such strategies on the energy and angular distribution of the species would also help predict and understand the characteristics of the final coating. Deposition parameters (e.g. mask shape, sample orientation, rotation speed, pressure, etc.) could be swept in silico over a wide range of values with a minimal need for experiments.

In this work, we present a multiscale modelling tool developed to predict film thickness at any point of any part, either static or in motion, subjected to a flux of material like in magnetron sputtering deposition. Because low pressure plasma deposition methods involve multiple collisions of the sputtered species, algorithm-based geometrical considerations like ray-tracing methods cannot be used. Instead, we combine Monte-Carlo transport algorithms with a collision-based softsphere model and space meshing techniques to predict matter deposition with the minimum computer resources (e.g. within a day). Special care is taken to optimise the code performance. The current version deals with neutral species only, and comparison with experimental data is performed for magnetron sputtering deposition of metal on a complex part in a large 3D coater.

In the first section of this article, the device, substrates and details of the experiments implemented to benchmark the code are presented. The second section is devoted to the modelling. It describes the management of complex meshed substrates, space cellularisation and the transport model. In the third and final section, the computed film thickness uniformity of chromium, silver and tantalum deposited on a complex substrate in static and dynamic mode is discussed in detail for three different pressures and compared with the experimental data. Later in the section, we present and discuss the film thickness and average energy by particle prediction of $\mathrm{Cr}$ deposition on parts in motion along one or two axis of rotation. Detailed information about the

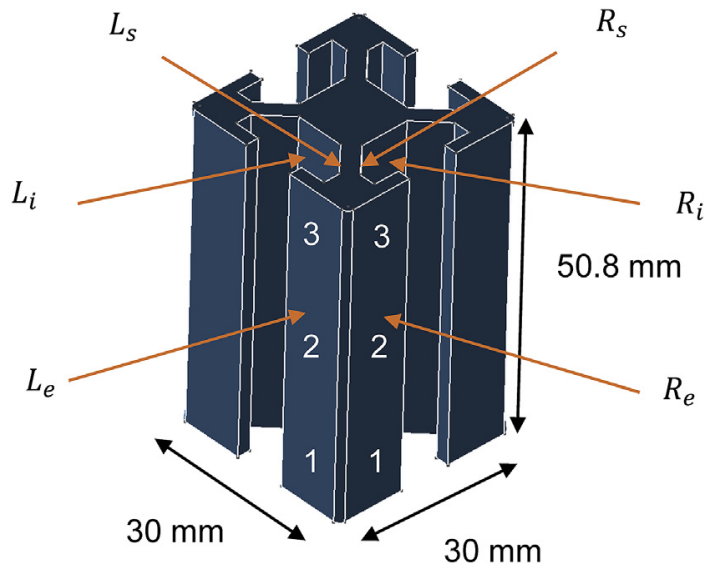

Fig. 1. Substrate description.

code and algorithm optimisations is given in the "supplementary materials" section.

\section{Experimental details}

\subsection{Substrate description}

The substrate is a standard commercial aluminium alloy profile (Fig. 1). Its main interest is to offer the possibility to study deposition on faces with different levels of shadowing. Three kinds of faces are distinguished with respect to their exposition to the sputtering source: fully exposed faces (index " $e$ "); inner faces (index " $i$ ") for those exposed and collimated by the two external lips; shadowed faces (index "s") on the inner structural elements. For each face, three regions are defined along the $\mathrm{z}$ axis (1, 2 and 3). Because of symmetry, only two sets of faces are considered in the static mode: $L$-faces (for Left) and $R$-faces (for Right).

In static mode, there are six different conditions classified according to their level of shadowing:

- $L_{e}$ parallel to the cathode and fully exposed,

- $L_{i}$ parallel to the cathode and collimated by the two lips,

- $L_{s}$ inclined with an angle of $45^{\circ}$, collimated by the two lips, but oriented toward the cathode,

- $R_{e}$ perpendicular to the cathode and fully exposed,

- $R_{i}$ perpendicular to the cathode and collimated by the two lips,

- $R_{S}$ inclined with an angle of $45^{\circ}$, collimated by the two lips, but oriented toward the inside of the chamber.

In dynamic mode, the rotation of the substrate enables to merge the $\mathrm{L}$ - and R-faces. In this paper, after experimental validation, only the Lfaces are considered.

\subsection{Experimental set-up}

The magnetron sputtering system (manufactured by Orange thin films, NL) used for this work is described in Fig. 2. It consists of a 319.51 hexagonal chamber where a planar rectangular cathode (dimensions: $75 \mathrm{~mm}$ by $350 \mathrm{~mm}$ ) is placed vertically on the right side (A). It is pumped with a $1450 \mathrm{l} / \mathrm{s}$ turbomolecular pump and pressure control is performed with a gas throttle valve. Gas is injected in the chamber through pierced tubes placed in the cathode vicinity (B). The substrate is fixed on a holder, which axis is at $160 \mathrm{~mm}$ from the cathode. The substrate centre is at a $40 \mathrm{~mm}$ height with respect to the cathode centre. In static mode, the $L_{e}$ face is kept parallel to the cathode surface.

In dynamic mode, two cases were considered: 


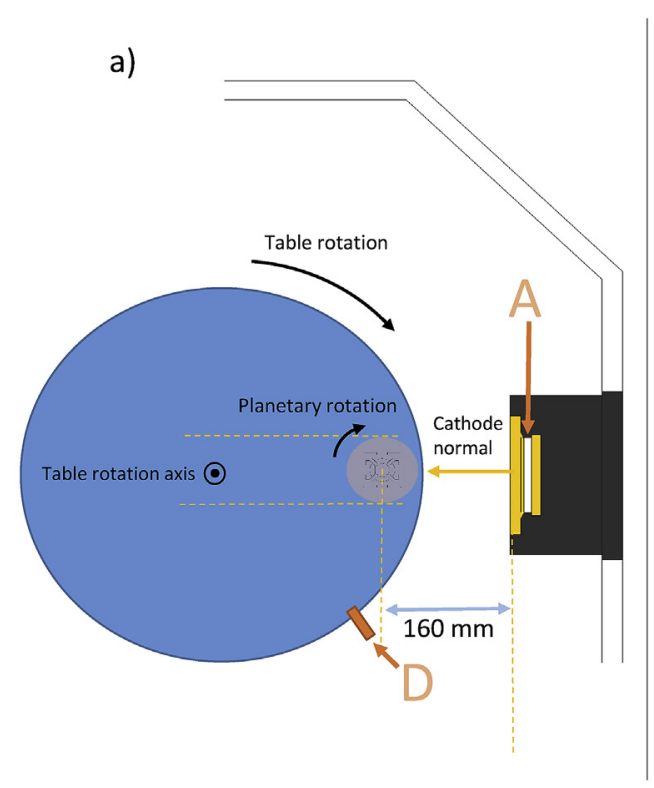

b)

Fig. 2. Experimental set-up of $\mathrm{Cr}, \mathrm{Ag}$ and $\mathrm{Ta}$ deposition on the complex substrate. 1) Top view, 2) lateral view.

- The one-fold rotation case, where the substrate rotates continuously on its symmetry axis (planetary rotation), but always in front of the cathode.

- The two-fold rotation case, where the table (in blue in Fig. 2) rotates continuously and the substrate rotates by $1 / 7$ th of a turn around the planetary axis thanks to a kicker (D) for each table rotation.

A DC power supply is used and the magnetic configuration of the cathode is balanced. The substrate is at a floating potential. The target racetrack measured with a $3 \mathrm{D}$ coordinate measuring machine (HEXAGON Manufacturing Intelligence DEA Global advantage) is shown on the right of Fig. 2 (c).

Deposition was performed with three metals of different atomic mass (Cr: $52 \mathrm{u}, \mathrm{Ag}: 108 \mathrm{u}$ and Ta: $181 \mathrm{u}$ ). Ar is injected at $150 \mathrm{sccm}$ and three pressures were investigated: $0.27,0.67$ and $1.3 \mathrm{~Pa}$. Table 1 resumes the experimental parameters for the static case.

Thickness measurements were performed by step edge profilometry with a DekTak 8 contact profiler ( $5 \mu \mathrm{m}$ stylus tip radius). The $L_{e}$ face is used as a reference for the deposition speed, as it is the maximal value.

For each sample exposition mode, three silicon samples were fixed on each face of interest (position 1, 2, 3 in Fig. 1) and partially masked with adhesive tape for film thickness measurements. For each set of deposition, 12 measurements were averaged on each of the 6 faces.

\section{Modelling}

The algorithm is described in Fig. 3:

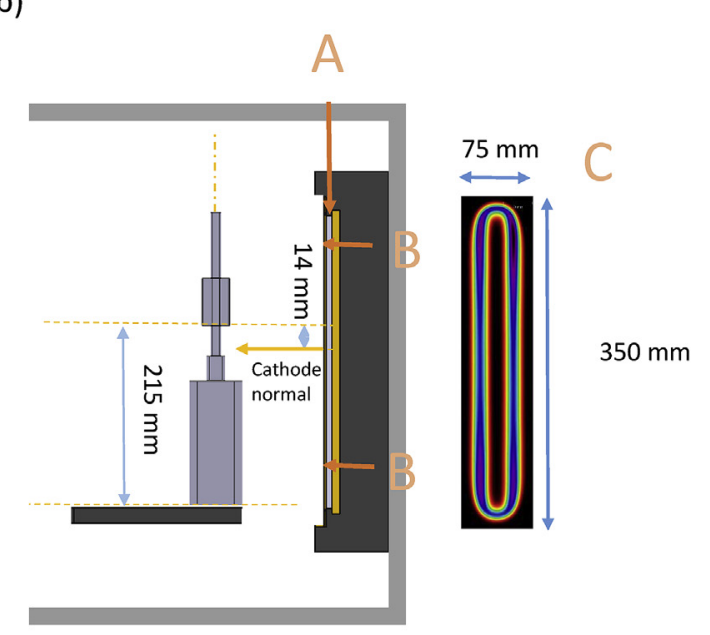

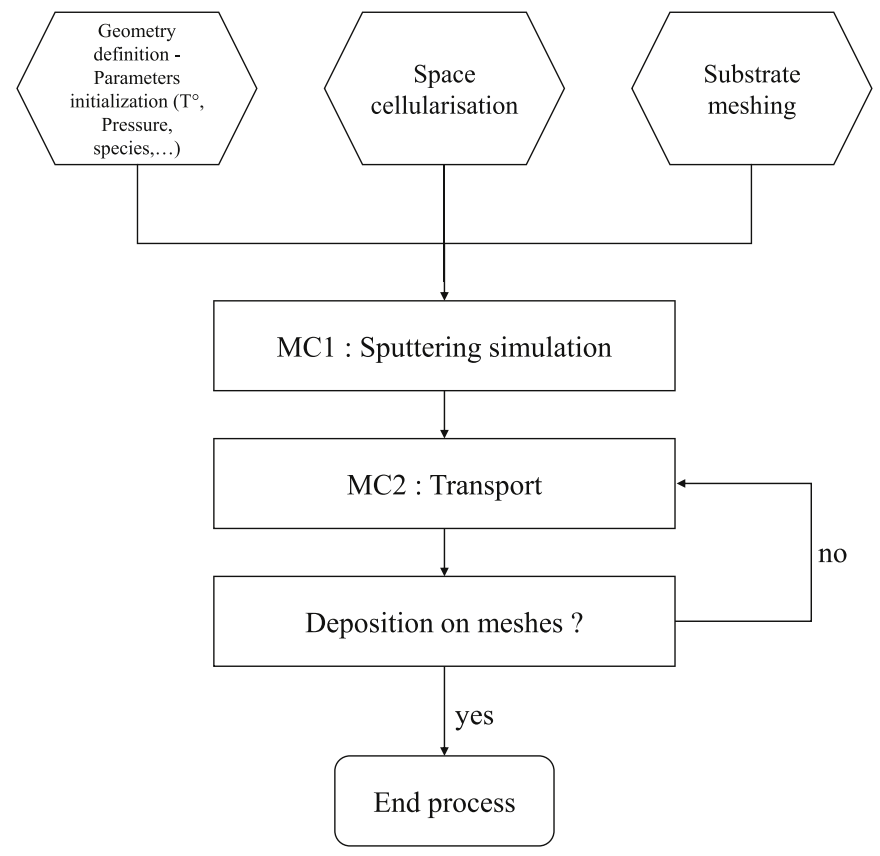

Fig. 3. Main structure of the algorithm.

a A 3D CAD/CAM representation of the chamber including all the objects that can interact with the deposited species is generated. It includes shutters, the targets, the substrate holder, shielding and

Table 1

Experimental parameters for the static case

\begin{tabular}{|c|c|c|c|c|c|c|}
\hline Cathode composition & Pressure $(\mathrm{Pa})$ & Current (A) & Voltage (V) & Power (W) & Deposition time (s) & Deposition rate on the $L_{e}$ sample $(\mu \mathrm{m} / \mathrm{h})$ \\
\hline $\mathrm{Cr}$ & 0.27 & 2 & 446 & 888 & 1800 & 4.18 \\
\hline $\mathrm{Cr}$ & 0.67 & 2 & 600 & 1200 & 2400 & 3.01 \\
\hline $\mathrm{Cr}$ & 1.3 & 2 & 546 & 1080 & 2400 & 3.82 \\
\hline $\mathrm{Ag}$ & 0.27 & 0.8 & 615 & 500 & 1800 & 8 \\
\hline $\mathrm{Ag}$ & 0.67 & 0.9 & 543 & 500 & 1800 & 8.2 \\
\hline $\mathrm{Ag}$ & 1.3 & 1 & 499 & 500 & 1800 & 5.6 \\
\hline $\mathrm{Ta}$ & 0.27 & 2 & 484 & 954 & 3500 & 3.7 \\
\hline $\mathrm{Ta}$ & 0.67 & 2 & 630 & 1256 & 3500 & 3.6 \\
\hline Тa & 1.3 & 2 & 543 & 1080 & 2700 & 4 \\
\hline
\end{tabular}




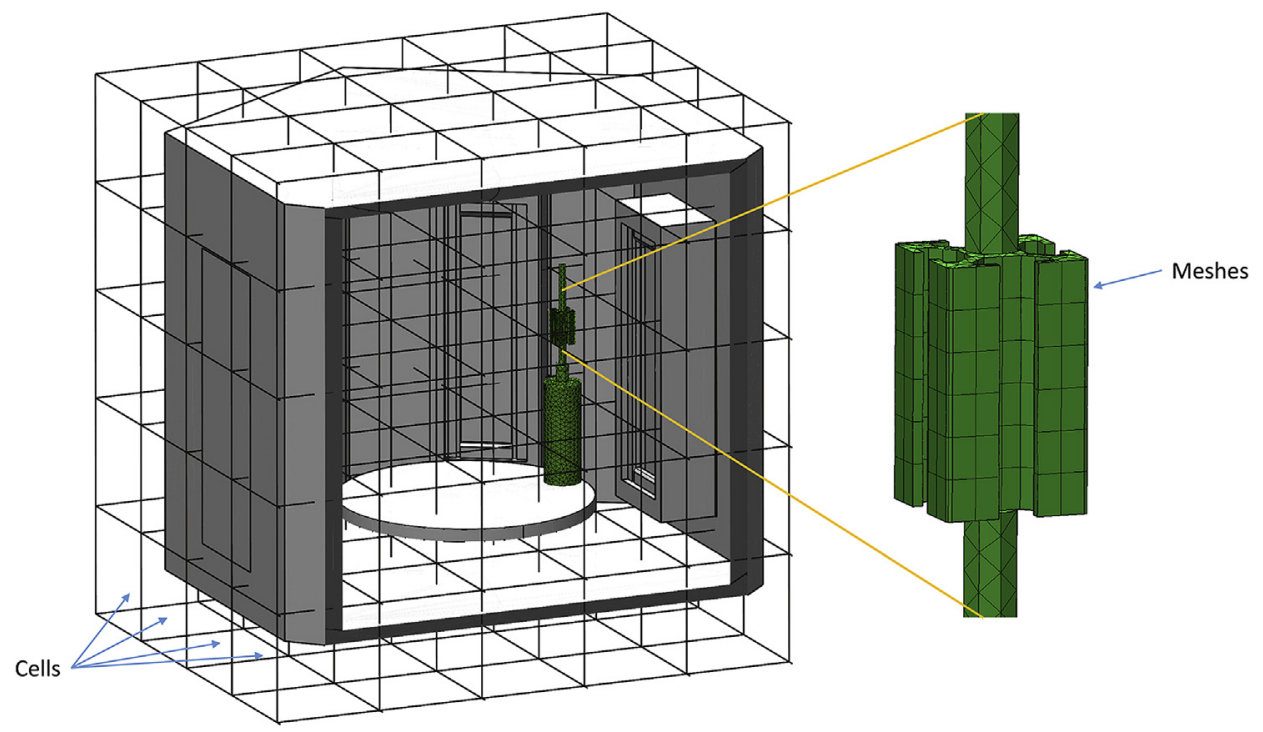

Fig. 4. Meshing of substrate and space cellularisation.

parts. Special care is taken for the target description since the racetrack topology needs to be defined [21]. In this study, we used a 3D scan of the target surface. But it can also be analytically described [22] or obtained by simulation [23]. In addition, the process simulation parameters based on the experimental data are initialized: temperature, pressure, cathode voltage, target composition, etc.

b The substrate surface as well as the space between the cathode and the sample table are meshed. In the following, the term "mesh" is used for the substrate modelling (2D meshes) and the term "cell" is used for the space divisions (3D cells).

c A first Monte Carlo simulation is performed with SRIM to compute the ejection of species from the cathode [24].

d A second Monte Carlo code simulates the transport of the metallic species all over the chamber by taking gas phase collisions into account.

e As the species progress, the algorithms test if the particles trajectories cross one or more substrate meshes, and determine on which mesh a particle will condense.

f The whole simulation ends when all particles are either deposited, or have performed more than a given number of collisions [13] (user input; 1200 in this work). In this latter case, the particle is deleted. $\mathrm{g}$ In dynamic mode, the steps $\mathrm{d}$. to f. are repeated whenever required.

One of the main features of this approach is to investigate the macroscopic shadowing of sample concavities or of other objects present in the chamber. Macroscopic shadowing is taken into account directly in the transport model. However, film growth is not implemented here. As a result, the ballistic shadowing or the nanometric sample surface topography $[25,26]$ (steps structures, corner coverages, patterned substrates) are not described. Nevertheless, outputs of the algorithm (as the energy and angular distribution at the sample surface) can be used in the future to simulate local film growth.

This algorithm combination computes relative deposition rate, deposited energy and angle, on any point of any substrate in motion in the vacuum chamber. When compared with the Direct Simulation Monte Carlo method (DSMC) [27], the main difference is the reduced computational cost since our simulations do not include the motion of every gas particle, but instead, consider the gas as a continuous entity defined by physical parameters like pressure and temperature. Indeed, in DSMC, each specie is represented by a great number of super-particles. Space is divided in cells for the purpose of reducing the number of partners in collisions and to compute the partial pressure, mean velocity, etc. at each time step, with high accuracy. The metal particles flux can be defined as a source of particles, which spread through the chamber and collide with the gas particles. This method, which requires high computer resources, can be used to study film deposition with the help of a surface model based on sticking coefficients. Reactive sputtering simulations are also possible with a more advanced surface model and can give more detailed results than the traditional "Bergmodel" [28]. It is possible to simulate moving substrates with the DSMC simulation approach but at high computational costs since one full simulation for every sample position would be required [29]. In the algorithm combination presented in this work, only metal atoms transport is simulated, to the benefit of computational resources when simulations with moving substrates are of interest.

Other codes use the same transport strategy but without space cellularisation [21,30-33]. In this case, the number of operations needed to test the intersection of particles with all substrate faces is tedious and time consuming. In our work, the use of cellularisation decreases the number of tests at each step and speeds up the computation (see the "supplementary materials" section).

\subsection{Volume and substrate description}

Each object surface (sample, shutters ...) is meshed with small independent planar triangles or parallelograms. A single mesh is defined by its contour and a vector normal to its surface, specifying his orientation (external or internal) with respect to the object volume. A physical object is defined by a closed set of meshes oriented outwards. Consequently, deposition happens only on the external side of each mesh, making possible to take the shadowing physical effect into account.

The internal volume of the coater is meshed with regular 3D cuboid elements (Fig. 4) with the aim to implement a "Verlet cells structure" [34]. Cell size is optimised to get the smaller simulation time (as described in "supplementary materials").

\subsection{Sputtering and transport simulation}

The target sputtering simulation is performed by SRIM [24] according to the average cathode sheath voltage, sputtering gas, target composition and surface binding energy. Ions are considered as accelerated though the sheath with a $0^{\circ}$ angle, i.e. perpendicular to the surface. This computation provides the initial angular and energy distribution of the sputtered particles at a very local scale. 
Particle emission from the target (at a macroscopic scale) is then simulated by a random position with a probability modulated by the racetrack depth according to a procedure already described by Mahieu et al. [21].

Transport in the gas phase happens with collisions between metal and gas species. The species interactions are generally described in terms of interatomic potential, which includes an attractive and a repulsive part. Energetic atoms such as the ones involved in sputter deposition are not that much subject to the attractive part, but mostly to the repulsive one (negative slope of the repulsive part of the potential). A screened-coulomb potential model is often used [30,31,35], and can accurately model scattering but to the expense of computational time. In rarefied gas flow modelling, these models are often replaced by computationally more efficient phenomenological models of a binary collision. This is especially true when the total number of surfaces of interest is high or when multiple simulations are required like for substrates in motion. In our work, we used a soft-shere potential as described by J. Geiser and S. Blankenburg [36]. The main difference between the potential and the soft-sphere approaches is that the former requires the calculation of an improper integral at each collision. On the contrary, the soft-sphere model has the advantage of using the trigonometric relations of hard sphere binary collisions but the cross-section is computed according to the value of the incident particle energy (see below). On the opposite, a pure hard sphere model makes the assumption of an easy-to- compute geometrical cross-section but does not describe the penetration of particles through the gas phase, namely the diffusion.

In this work, the collisions are computed in the following way. The particles travel in straight line between two collisions along a distance randomly generated by $\lambda=-\lambda_{m} \ln \omega$, with $\omega$ generated from a uniform distribution $U(] 0,1[)$, and $\lambda_{m}$ is the mean free path:

$\lambda_{m}=\frac{k_{B} T}{\sigma(s) P_{g a s}}$

where $s$ is the reduced energy: $s=\left|v_{\text {metal }}\right| \sqrt{\frac{m_{g}}{2 k_{B} T}}$.

In order to compute the collisional cross section, two cases are considered [30] using $E=\frac{3 k_{B} T}{2}$ as a criterion $(\mathrm{E}=0.0387 \mathrm{eV}$ at $300 \mathrm{~K})$ :

- $\operatorname{If}\left(E>\frac{3 k_{B} T}{2}\right)$, the particle has enough energy to consider the gas as motionless. It has a penetration which is modelled by:

$\sigma(s)=\sigma_{0}\left(\frac{\left[\left(s+\frac{1}{2 s}\right) \operatorname{erf}(s)+\frac{1}{\sqrt{\pi}} \exp \left(-s^{2}\right)\right]}{3 s}\right)$.

where $s$ has been defined above, $\sigma_{0}$ is the geometrical cross-section:

$\sigma_{0}=\pi\left(r_{s}+r_{g}\right)^{2}\left(1+\frac{m_{s}}{m_{g}}\right)^{\frac{1}{2}}$,

$m_{g}$ is the atomic mass of the gas atom, $m_{s}$ the atomic masse of the sputtered specie and $r_{i}$ is the van der Waals radius of the speciei.

After each collision, the particle loss energy is computed as:

$E_{\text {out }}=E_{\text {in }}\left(1-\frac{2\left(1-\cos \left(\theta_{\text {com }}\right)\right) \mu}{m_{s}+m_{g}}\right)$.

where $\mu$ designs the reduced mass of the system $\left(\mu=\frac{m_{s} m_{g}}{m_{s}+m_{g}}\right)$ and the scattering angle in the system centre of mass is modelled by:

$\theta_{\text {com }}=2 \operatorname{acos}(z)$,

with $z \in U[0,1]$. Notice that in the laboratory system, the angle is a function of the gas and particle sputtered masses.

- $\operatorname{If}\left(E \leq \frac{3 k_{B} T}{2}\right)$, the particle is considered as thermalized and the motion is assumed as a random walk. The cross-section is $\sigma(s)=\sigma_{0}$, the energy is set to $=\frac{3 k_{B} T}{2}$ and scattering is assumed as isotropic [37]:

$\theta_{l a b}=\operatorname{acos}(2 z-1)$

\subsection{Deposition on meshes}

For each particle and between two collisions, the intersection between the particle trajectory and a mesh is investigated according to the algorithm developed by J. J. Jiménez et al. [38]. If the test results are "true", the particle is deposited on the mesh. Otherwise, the particle continues its trajectory and a new collision is computed [32,39,40]. In order to reduce the computation time, this algorithm was complemented with a modified version of a cell-linked list method [41], which consists in checking the intersection only in a small volume close to the mesh. More details are given in "supplementary materials".

The sticking coefficient is set to one, which means that no backscattering is allowed and the film density is supposed constant and maximum, since no porosity is computed. Thickness is then defined by:

Thickness $=\frac{N_{\text {particle }}}{S_{\text {mesh }}}$.

where $N_{\text {particle }}$ is the total number of deposited particles on a mesh and $S_{\text {mesh }}$ is the surface of the mesh.

\subsection{Simulation parameters}

The simulation parameters are listed in Table 2.

Each substrate mesh on which deposition occurs is of the parallelogram type, and presents a surface of around $40 \mathrm{~mm}^{2}$. Each experimental silicon sample is therefore described by 4 meshes and 12 meshes describe a face. The 3D cuboid elements have a cell edge of about $40 \mathrm{~mm}$ and a volume of $64 \mathrm{~cm}^{3}$. Temperature is set at $300 \mathrm{~K}$.

In accordance with formulas 3.1 and 3.2, the cross-section increases with the atoms diameter and the mean free path decreases with the increase of the pressure for each atom type. Here, only the collisions between the sputtered specie and gas atoms are considered. The mean free path also decreases with the increase of the species mass.

For each simulation, 5 million atoms were launched from the target. Simulations, performed on an Intel ${ }^{\circledast}$ Core $^{\mathrm{TM}}$ i7-7820X CPU $3.6 \mathrm{GHz}$, last approximatively $30 \mathrm{~min}$ (unparalleled $\mathrm{C}++$ code). Generally, the lower the pressure, the faster the simulation. Indeed, it is obvious that, at low pressure, $\lambda_{m}$ is large and consequently, for a fixed target-tosubstrate distance, less collisions happen, which means less computations.

\section{Results and discussion}

\subsection{Static case}

Fig. 5 shows the correlation between the simulation and experimental results for $\mathrm{Cr}, \mathrm{Ag}$ and $\mathrm{Ta}$ depositions at the three different

Table 2

Simulation parameters

\begin{tabular}{llllll}
\hline $\begin{array}{l}\text { Cathode } \\
\text { composition }\end{array}$ & Pressure $(\mathrm{Pa})$ & $\sigma_{0}\left(\mathrm{~nm}^{2}\right)$ & $\lambda_{0}(\mathrm{~mm})$ & $\begin{array}{l}\text { Sputtering } \\
\text { yield (SRIM } \\
\text { calculation) }\end{array}$ & $\begin{array}{l}\text { Number of } \\
\text { atoms } \\
\text { sputtered }\end{array}$ \\
\hline $\mathrm{Cr}$ & 0.27 & 0.59 & 26.0 & 1.25 & $510^{6}$ \\
$\mathrm{Cr}$ & 0.67 & 0.59 & 10.4 & 1.25 & $510^{6}$ \\
$\mathrm{Cr}$ & 1.33 & 0.59 & 5.2 & 1.25 & $510^{6}$ \\
$\mathrm{Ag}$ & 0.27 & 0.78 & 19.8 & 2.17 & $510^{6}$ \\
$\mathrm{Ag}$ & 0.67 & 0.78 & 7.9 & 2.17 & $510^{6}$ \\
$\mathrm{Ag}$ & 1.33 & 0.78 & 3.9 & 2.17 & $510^{6}$ \\
$\mathrm{Ta}$ & 0.27 & 1.2 & 12.6 & 0.85 & $510^{6}$ \\
$\mathrm{Ta}$ & 0.67 & 1.2 & 5.1 & 0.85 & $510^{6}$ \\
$\mathrm{Ta}$ & 1.33 & 1.2 & 2.5 & 0.85 & $510^{6}$ \\
\hline
\end{tabular}



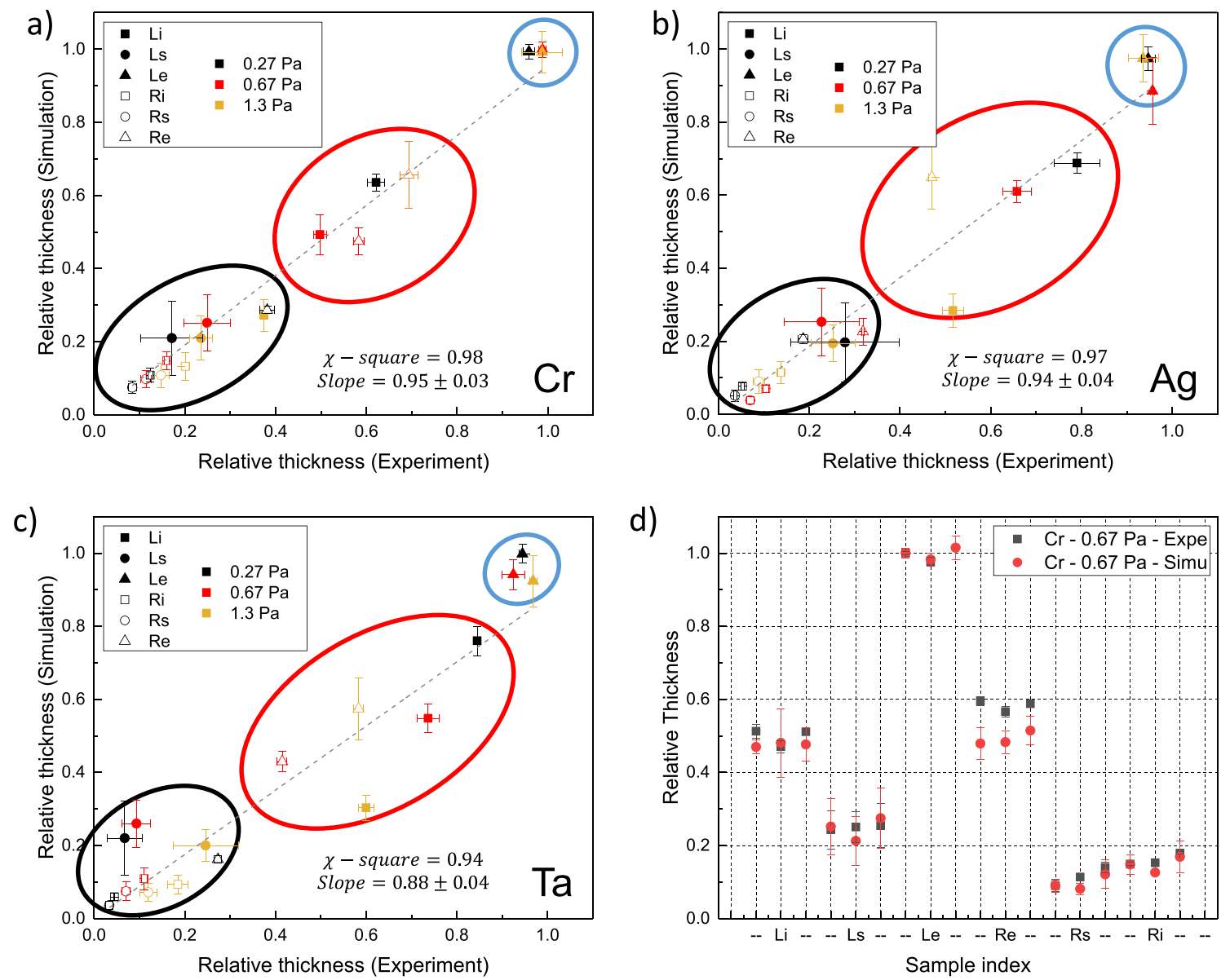

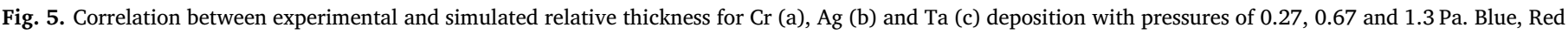

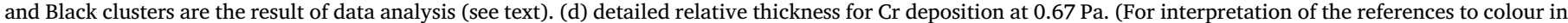
this figure legend, the reader is referred to the Web version of this article.)

pressures. Because the film thickness difference between the three silicon samples of each surface was lower than $5 \%$, each experimental data corresponds to an average value of the three film thicknesses. Consequently, the film thickness was computed by averaging 12 meshes for the simulated data. The relative thickness of the sample $i\left(t_{r i}\right)$ is normalized as:

$t_{r i}=\frac{t_{i}}{t_{L_{e}}}$

A global analysis of Fig. 5 a, b, c, reveals a general good agreement between the simulations and the experiments. Whatever the material, the face or the pressure, the slope of the linear fit is near 1 and $\chi^{2}$ is higher than 0.94. In a large range of situations, the film thickness is predictable within about $10 \%$ error, which is comparable to an experimental error and completely acceptable. Second, the lighter the element, the better the estimation of the local thickness, and the lower the pressure, the better the prediction. The first reason lies in the choice of the soft sphere potential model. In this model, the scattering angle is computed according to equation (3.5), which is independent of the energy. The assumption is not fully valid because, when a particle is non-thermalized, a dipole-effect appears and the scattering angle becomes Z-dependent (nucleus charge dependent) and is not species independent anymore. Moreover, the larger the $\mathrm{Z}$, the higher the dipole effect. Especially with energetic particles, with heavy energetic particles, a screened-coulomb potential should then give more precise results if needed. Concerning the pressure dependence, the fact that equation (3.6) does not take into account film density plays a big role in the thickness prediction. At higher pressure, the number of particles condensing on the substrate with a low energy is very large (see Fig. 6 below). It is supposed to form films with more porosity.

Results analysis enabled us to cluster most of the exposed faces data ( $\mathrm{L}$ or $\mathrm{R}$ ), whatever the material or pressure. Indeed, the black cluster (low thicknesses) corresponds to the less exposed faces $\left(R_{s}, R_{i}\right.$ and $\left.L_{s}\right)$, while the blue cluster corresponds to the most exposed faces $\left(L_{e}\right)$. The red larger cluster contains data for only the $L_{i}$ faces. $L_{i}$ is parallel to the cathode, but not fully exposed because of the lips. A special case is the $R_{e}$ face (tilted at $90^{\circ}$ with respect to the cathode), which is pressure dependant: $R_{e}$ appears in the blue cluster for $0.67 \mathrm{~Pa}$ and $1.3 \mathrm{~Pa}$, but belongs to the black cluster for $0.27 \mathrm{~Pa}$. This is seen for all the material tested here. It is due to the scattering during transport. Indeed, for low pressure, only a small amount of particles is thermalized and can reach the substrate. The film thickness is then small. At higher pressure, this contribution is higher, and leads to relatively bigger films.

Species condensing on the faces in the blue cluster (most exposed faces) have a higher directionality, while species belonging to the black cluster (least exposed surfaces) have a lower directionality. This is related to the number of collisions the species undergo, which in turn affect the average energy per particle condensing on the faces. It is described in Fig. 6, where the results of the average energy modelling of the particles deposited per face are presented.

As expected, for each face, one can see that the lower the pressure, the higher the energy. Also, the average energy of arriving particles is material dependent: Ta atoms condense on the substrate with a generally higher energy that $\mathrm{Cr}$ and $\mathrm{Ag}$ because of higher ejection energy from the cathode and despite a higher energy loss as shown in Table 3.

By comparing the trend in between the faces, one can see that the 
a)

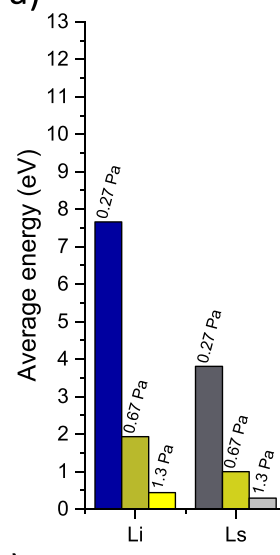

c)

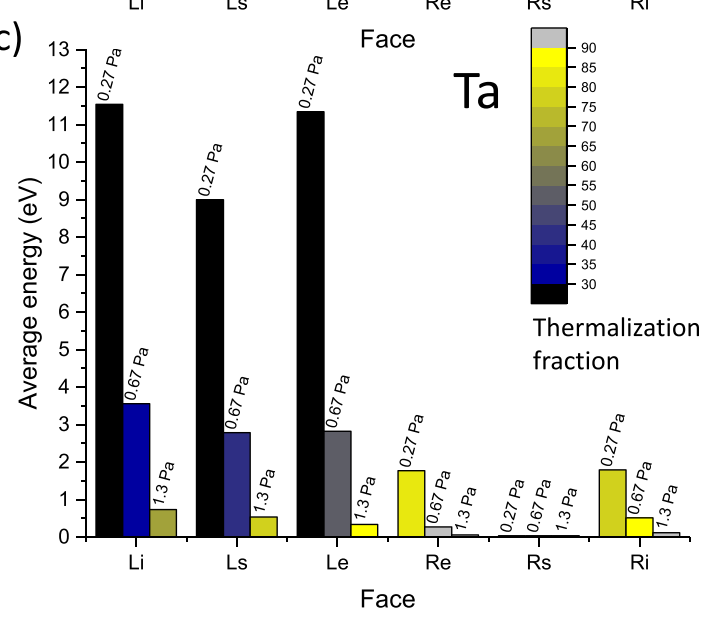

b)

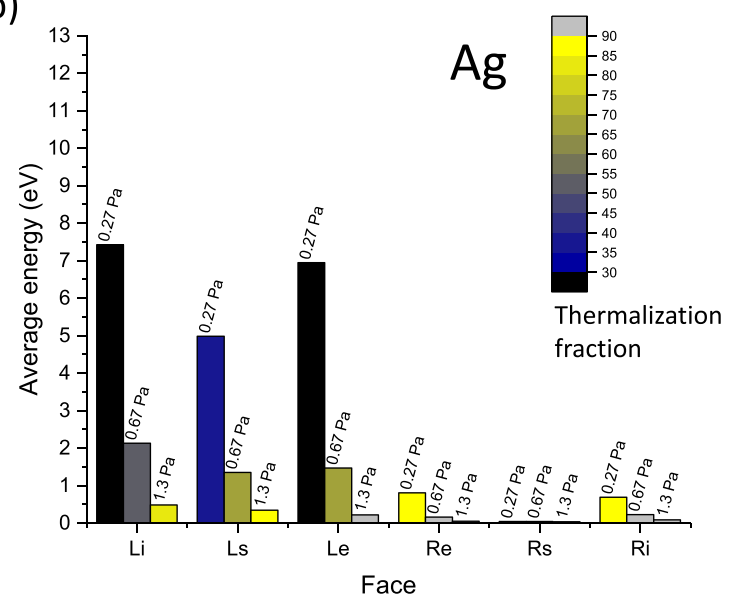

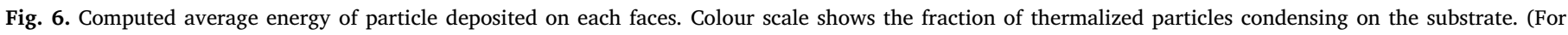
interpretation of the references to colour in this figure legend, the reader is referred to the Web version of this article.)

Table 3

modelled average ejection energy, energy loss and relative loss

\begin{tabular}{lllll}
\hline $\begin{array}{l}\text { Cathode } \\
\text { composition }\end{array}$ & Pressure (Pa) & $\begin{array}{l}\text { Average } \\
\text { ejection } \\
\text { energy }(\mathrm{eV} / \\
\text { part) }\end{array}$ & $\begin{array}{l}\text { Average } \\
\text { energy by } \\
\text { particle }(\mathrm{eV} / \\
\text { part) }\end{array}$ & $\begin{array}{l}\text { Energy loss } \\
\text { in gas phase } \\
(\%)\end{array}$ \\
\hline $\mathrm{Cr}$ & 0.27 & 24.7 & 6.3 & 74 \\
$\mathrm{Cr}$ & 0.67 & 24.7 & 4.2 & 83 \\
$\mathrm{Cr}$ & 1.33 & 24.7 & 2.2 & 91 \\
$\mathrm{Ag}$ & 0.27 & 18.5 & 3.5 & 81 \\
$\mathrm{Ag}$ & 0.67 & 18.5 & 0.6 & 97 \\
$\mathrm{Ag}$ & 1.33 & 18.5 & 0.1 & 99 \\
$\mathrm{Ta}$ & 0.27 & 30.8 & 6.7 & 78 \\
$\mathrm{Ta}$ & 0.67 & 30.8 & 1.3 & 95 \\
$\mathrm{Ta}$ & 1.33 & 30.8 & 0.2 & 99 \\
\hline
\end{tabular}

less the face is exposed, the lower the average energy and the larger the thermalization fraction. This is especially true for the $R_{S}$ face, where almost $100 \%$ of the particles are thermalized whatever the specie or the pressure. This is expected since a high number of collisions are performed by the species before reaching this surface. The object complexity produces a high shadowing on this surface. For the more exposed faces $\left(L_{e}, L_{i}, L_{s}\right)$ at low pressure, the thermalization ratio is globally lower than $40 \%$. The more the metal is heavy, the more this fraction is low (less than $20 \%$ for Ta). Generally, the average energy is in invert relation with the thermalization ratio.

One has to note that the average energy per particle condensing on the partly shadowed $L_{i}$ face is larger than the one condensing on the $L_{e}$ face, even though both are parallel to the cathode surface. This is due to a shadowing effect of the two lips acting as a collimator, which only lets the highly energetic and directional particles pass. An inverse phenomenon happens for the highly shadowed $R_{s}$ face on which only the particles encountering a significant number of collisions condense. Despite the absence of shadowing, the $R_{e}$ surface has a very high thermalization fraction. This effect is obviously related to the surface orientation of $90^{\circ}$ compared to the target surface. Only the particles which perform a large number of collisions, and consequently lose their initial energy completely, condense on this surface.

In terms of angular exposition, all the faces parallel to each other have particle condensing with the same pattern of energy per particle $\left(R_{e}\right.$ and $R_{i}, L_{e}$ and $L_{i}$ ). Particles condensing on faces perpendicular to each other have a different energy pattern and give rise to a different morphological coating. Indeed, while the $L_{e}$ face is parallel to the cathode surface and produces vertically aligned microstructures, the $R_{e}$ face is in a Glancing Angle Deposition (GLAD) condition and produces tilted microstructures as illustrated on the SEM micrographs shown in Fig. 7 for $\mathrm{Cr}$ deposition at $0.27 \mathrm{~Pa}$.

On the $R_{e}$ face, the film morphology is columnar with tilted columns and secondary growth, while for the $L_{e}$ face, the morphology is denser with no tilted columns. This has already been reported in literature, especially for GLAD deposition, where the incident flux angle is related to the film density. The column tilt angle $\left(18^{\circ}\right)$ is in agreement with Tait's law $\left(18^{\circ}\right.$ with a resultant incident angle of $\left.19^{\circ}\right)$ [42,43]. GLAD deposition is known to produce less dense coatings, and this explains why the agreement between experiment and modelling of $\mathrm{Cr}$ deposition on the $R_{e}$ face is lower than for any other face (Fig. 5d): indeed, our modelling assumes a bulk elemental density, whereas real coatings deposited in GLAD mode exhibit substantial porosity and therefore a higher thickness.

As our modelling is validated by the static study case, one should 

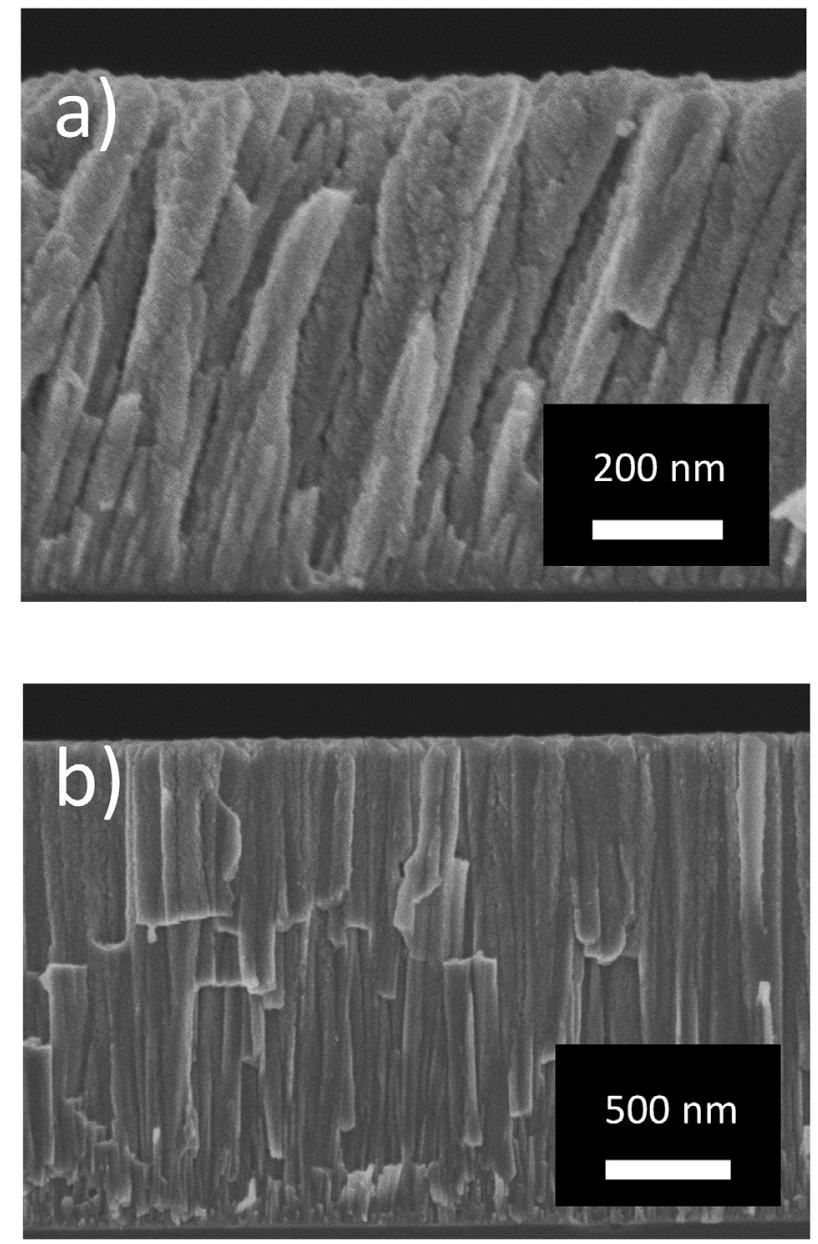

Fig. 7. Cross-section SEM pictures of $\mathrm{Cr}$ films deposited at $0.27 \mathrm{~Pa}$ for the samples $R_{e}$ (a) and $L_{e}$ (b).

investigate more realistic and complex deposition cases with the use of substrate rotation.

\subsection{Dynamic case}

In this section, one-fold and two-fold rotations are studied for chromium for the three previous pressures. Tantalum and silver give similar results. The rotation introduces a new symmetry in the system and thus enables to consider only the $L_{e}, L_{i}$ and $L_{s}$ faces. Fig. 8 compares the modelled film thickness with the experimental measurements.

Compared to the static case, the correlation between simulation and experiment is very good $\left(\chi^{2}>0.99\right.$ and slope close to 1$)$, and even better. Even in these complex configurations, an accurate prediction of the thickness is possible.

The effect of rotation is clearly noticeable in Fig. 8a and b: there are only two clusters. The blue one still corresponds to the $L_{e}$ face, which is the most exposed to the particle flux and with the shortest target to substrate distance. All the other shadowed faces belong to the black cluster. The latter is shifted towards higher values (higher thickness) compared to the static case because of the sample motion. No relative thicknesses lower than 0.2 are found, while it can reach 0.05 in the static case. The structural shadowing effect of the substrate is balanced by the rotation. Indeed, each face encounters more favourable positions and orientations in terms of flux exposure.

On the one hand, the use of rotation is successful: the global film thickness homogeneity on the substrate is improved, especially for the inner $L_{i}$, and $L_{s}$ faces, which are also thicker. On the other hand, the difference between the inner and outer faces is emphasized.
In terms of film thickness, no significant differences are clearly observed for one-fold or two-fold rotation. This is confirmed when looking at Fig. 8c and d, where the rotation only shows differences between the most exposed face $\left(L_{e}\right)$ and the others. One can only note that the two-fold rotation decreases the differences between the inner faces a little more. This result can be surprising since the common way to increase the thickness uniformity in the industry is using more rotation axis. M. Panjan discussed at length in Ref. [19] the influence of the gear ratios on the thickness deposited on a flat substrate. The addition of many rotation axis can indeed increase the thickness uniformity between the different parts loaded in the machine. Here, the point is that for one single part, the change from one to two-fold rotation does not change sensitively the uniformity between inner or shadowed $\left(L_{i}\right.$ and $\left.L_{s}\right)$ faces and exposed faces $\left(L_{e}\right)$. To generalize this conclusion, the same work must be realized for a wide range of gear ratios.

Fig. 9 presents the average energy deposited on the three studied faces for the previous static case (a), with the one-fold (b) and two-fold (c) rotation. First, a homogeneity effect is seen for the average energy and the thermalization fraction. For a given pressure and a given rotation, these values are very narrow whatever the face. Second, it is obvious that the average energy decreases with the complexity of the motion: it is approximately divided by two between static and one-fold rotation, and again between one-fold and two-fold rotation. This is directly related to the exposition time of each face to the particle flux. In static: the faces are exposed permanently. In one-fold rotation: the faces are exposed only during a part of a lap, which is even reduced for two-fold rotation. In addition, each face is exposed to a fully thermalized flux during most of the deposition time. In the best case, i.e. for low pressure, the thermalization fraction is higher than $60 \%$ in the onefold rotation case and higher than $80 \%$ in the double one. The effect of the pressure is still there and a strong decrease of the average energy combined with an increase of the thermalization fraction is observed.

It is worth to note that, if in terms of film thickness, the type of rotation (one-fold or two-fold) does not change significantly, in terms of energy, the differences are obvious. It means that the properties of the films are affected by the motion choice, even if the thickness criterion is validated.

\section{Conclusions}

In this work, we set up a computational model able to predict the thickness of films deposited by PVD on complex shapes in motion. Only neutrals are considered and collisions in the gas phase are described by a soft-sphere model. The geometrical parts are described by meshed surfaces and efforts were made to minimize the computational resources by implementing fast algorithms. Simulation predictions at three different pressures were compared with chromium, silver and tantalum experimental depositions on static and dynamic parts including shadowed and non-shadowed faces. Our modelling correctly reproduces all the experimental results, whatever the face, the deposited material, the pressure and the motion type.

The average energy and the thermalization ratio were investigated and the influence of shadowing and exposition to the atoms flux on the energy deposited was highlighted for two different rotation modes. The main result is that the rotation mode only influences the relative thickness of the shadowed faces and the global film thickness homogeneity on the substrate is improved. No significant difference was found between one-fold or two-fold rotation for the considered geometry.

On the contrary, our simulations reveal that the motion type has a strong influence on the average particle energy condensing on the substrate: the more the samples are subject to complex rotations, the more the energy of species condensing locally is thermalized. This observation may help to better understand the rotation effect on film morphology and opens the way for film properties prediction, e.g. stress 


\section{One-fold rotation}

a)

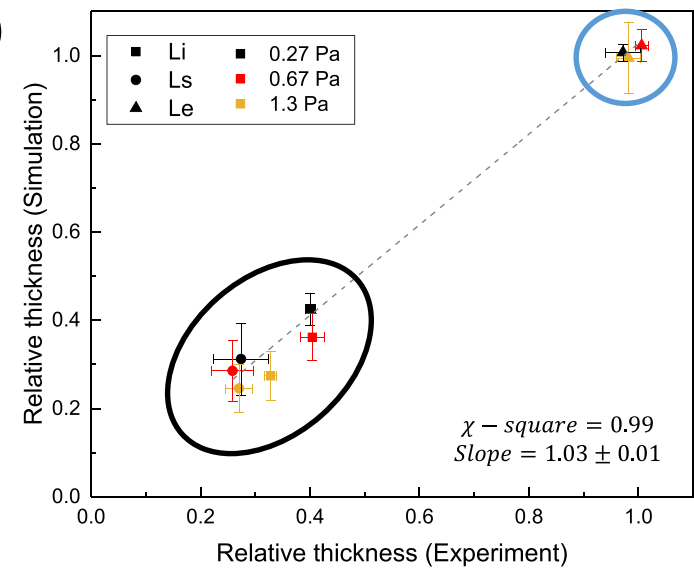

c)

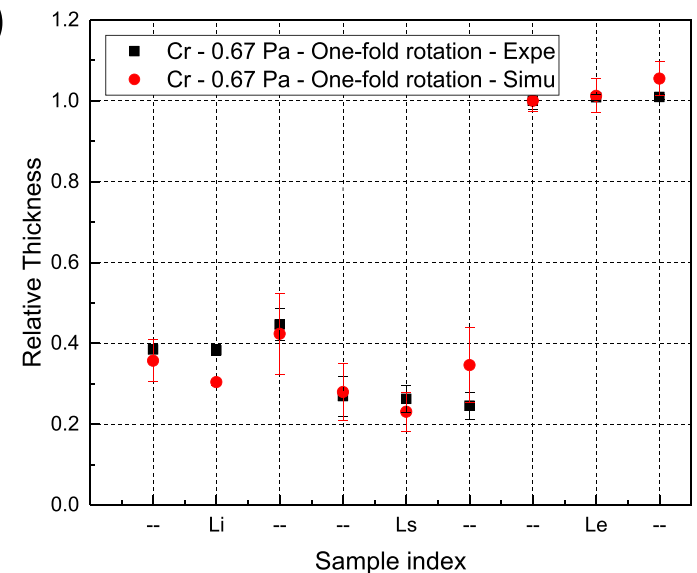

Two-fold rotation
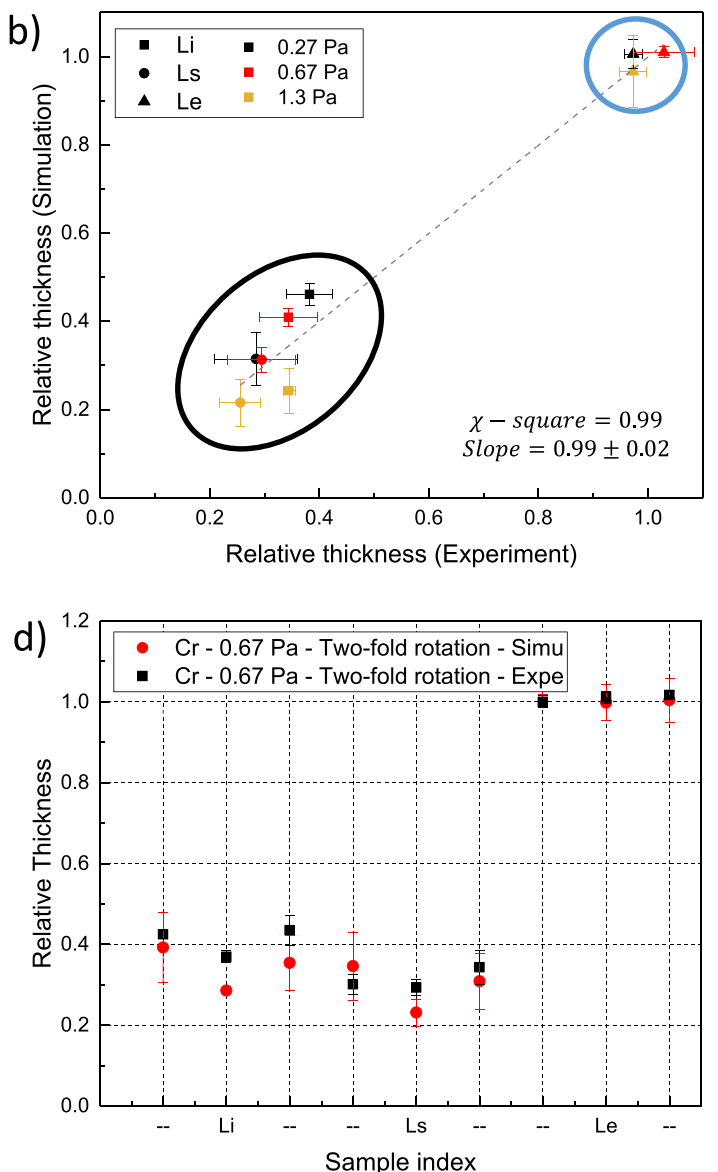

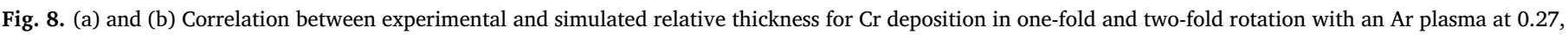
0.67 and 1.3 Pa. (c), (d): detailed relative thickness for $\mathrm{Cr}$ deposition in one-fold and two-fold rotation at $0.67 \mathrm{~Pa}$.
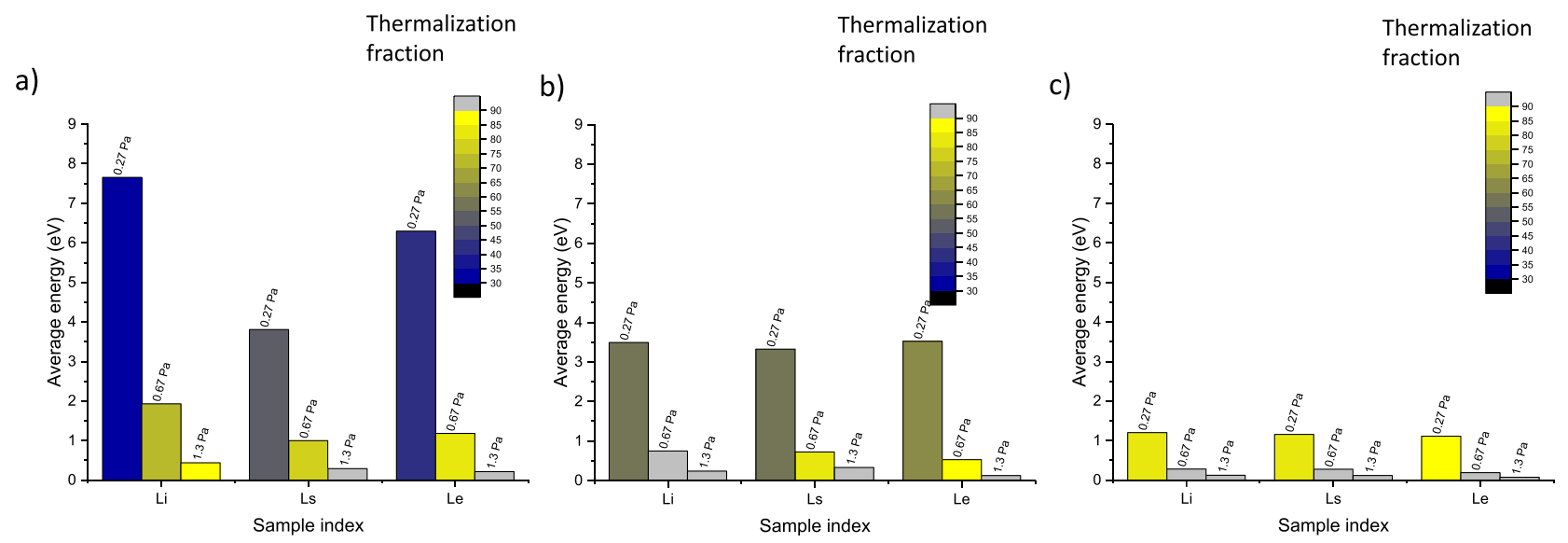

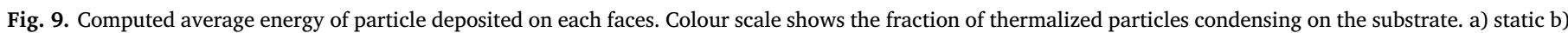

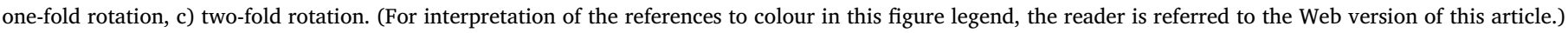

and mechanical performances.

\section{Acknowledgments}

The authors M. Evrard and S. Lucas gratefully acknowledge the financial support of the Walloon region under the FEDER and 3DCOATER-5: Convention $\mathrm{N}^{\circ} 1610258$, project 3215. The author A. Besnard also thanks the "Région Bourgogne Franche-Comté" for the concession of the grant (Rech-Mobi-000026).

\section{Appendix A. Supplementary data}

Supplementary data to this article can be found online at https:// doi.org/10.1016/j.surfcoat.2019.125070.

\section{References}

[1] J. Lu, M.J. Kushner, Trench filling by ionized metal physical vapor deposition, J. Vac. Sci. Technol. A Vacuum, Surfaces, Film 19 (2002) 2652-2663, https://doi.org/ 
10.1116/1.1399318

2] L. Pinard, C. Michel, B. Sassolas, L. Balzarini, J. Degallaix, V. Dolique, R. Flaminio, D. Forest, M. Granata, B. Lagrange, N. Straniero, J. Teillon, G. Cagnoli, Mirrors used in the LIGO interferometers for first detection of gravitational waves, Appl. Opt. 56 (2017) C11, https://doi.org/10.1364/ao.56.000c11.

[3] C.K. Madsen, J.H. Zhao, Optical Filter Design and Analysis (1999), https://doi.org/ 10.1002/0471213756.

[4] H.K. Raut, V.A. Ganesh, A.S. Nair, S. Ramakrishna, Anti-reflective coatings: a critical, in-depth review, Energy Environ. Sci. 4 (2011) 3779-3804, https://doi.org 10.1039/c1ee01297e.

[5] M. Lenner, A. Fiedler, C. Spielmann, Reliability of laser safety eye wear in the femtosecond regime, Opt. Express 12 (2004) 1329, https://doi.org/10.1364/opex. 12.001329.

[6] B. Wang, X. Fu, S. Song, H. Chu, D. Gibson, C. Li, Y. Shi, Z. Wu, B. Wang, X. Fu, S. Song, H.O. Chu, D. Gibson, C. Li, Y. Shi, Z. Wu, Simulation and optimization of film thickness uniformity in physical vapor deposition, Coatings 8 (2018) 325, https://doi.org/10.3390/coatings8090325.

[7] G. Abadias, E. Chason, J. Keckes, M. Sebastiani, G.B. Thompson, E. Barthel, G.L. Doll, C.E. Murray, C.H. Stoessel, L. Martinu, Review Article: stress in thin films and coatings: current status, challenges, and prospects, J. Vac. Sci. Technol. A Vacuum, Surfaces, Film. 36 (2018) 020801, https://doi.org/10.1116/1.5011790.

[8] A. Vickery, C.P. Jensen, F.E. Christensen, M.P. Steenstrup, Collimated magnetron sputter deposition for mirror coatings, X-Ray Opt. Instrum. 2008 (2008) 1-9, https://doi.org/10.1155/2008/792540.

[9] C. Liu, M. Kong, C. Guo, W. Gao, B. Li, Theoretical design of shadowing masks for uniform coatings on spherical substrates in planetary rotation systems, Opt. Express 20 (2012) 23790, https://doi.org/10.1364/oe 20.023790.

[10] B. Sassolas, R. Flaminio, J. Franc, C. Michel, J.-L. Montorio, N. Morgado, L. Pinard, Masking technique for coating thickness control on large and strongly curved aspherical optics, Appl. Opt. 48 (2009) 3760, https://doi.org/10.1364/ao.48.003760.

[11] J. Arkwright, I. Underhill, N. Pereira, M. Gross, Deterministic control of thin film thickness in physical vapor deposition systems using a multi-aperture mask, Opt. Express 13 (2005) 2731, https://doi.org/10.1364/opex.13.002731.

[12] K.S. Fancey, A coating thickness uniformity model for physical vapour deposition systems: further analysis and development, Surf. Coat. Technol. 105 (1998) 76-83, https://doi.org/10.1016/S0257-8972(98)00447-2.

[13] H. Adachi, K. Wasa, I. Kanno, H. Kotera, Handbook of Sputter Deposition Technology: Fundamentals and Applications for Functional Thin Films, NanoMaterials and MEMS, William Andrew, 2012.

[14] B. Rother, On the possibility of physical vapour deposition process design by coordinated substrate rotation modes, Surf. Coat. Technol. 64 (1994) 155-159, https://doi.org/10.1016/0257-8972(94)90102-3.

[15] B. Rother, G. Ebersbach, H.M. Gabriel, Substrate-rotation systems and productivity of industrial PVD processes, Surf. Coat. Technol. (1999), https://doi.org/10.1016/ S0257-8972(99)00120-6.

[16] M. Panjan, M. Čekada, P. Panjan, T. Peterman, Computer simulation of multilayer structure of TiAlN/CrN coatings, Plasma Process. Polym. 4 (2007) 921-926, https://doi.org/10.1002/ppap.200732206.

[17] M. Panjan, T. Peterman, M. Čekada, P. Panjan, Simulation of a multilayer structure in coatings prepared by magnetron sputtering, Surf. Coat. Technol. 204 (2009) 850-853, https://doi.org/10.1016/j.surfcoat.2009.08.026.

[18] M. Panjan, S. Šturm, P. Panjan, M. Čekada, The influence of rotation during sputtering on the stoichiometry of TiAlN/CrNx multilayer coating, Surf. Coat. Technol. 203 (2008) 554-557, https://doi.org/10.1016/j.surfcoat.2008.04.082.

[19] M. Panjan, Influence of substrate rotation and target arrangement on the periodicity and uniformity of layered coatings, Surf. Coat. Technol. 235 (2013) 32-44, https:// doi.org/10.1016/j.surfcoat.2013.06.126.

[20] C.M. Müller, A.S. Sologubenko, S.S.A. Gerstl, M.J. Süess, D. Courty, R. Spolenak, Nanoscale $\mathrm{Cu} / \mathrm{Ta}$ multilayer deposition by co-sputtering on a rotating substrate. Empirical model and experiment, Surf. Coat. Technol. 302 (2016) 284-292, https:// doi.org/10.1016/j.surfcoat.2016.06.024.

[21] K. Van Aeken, S. Mahieu, D. Depla, The metal flux from a rotating cylindrical magnetron: a Monte Carlo simulation, J. Phys. D Appl. Phys. 41 (2008), https://doi. org $/ 10.1088 / 0022-3727 / 41 / 20 / 205307$

[22] D. Depla, Magnetrons, Reactive Gases and Sputtering, (2014).

[23] S. Kadlec, Computer simulation of magnetron sputtering - experience from the industry, Surf. Coat. Technol. 202 (2007) 895-903, https://doi.org/10.1016/j. surfcoat.2007.06.043.

[24] J.F. Ziegler, J.P. Biersack, The stopping and range of ions in matter, Treatise HeavyIon Sci. 1985, pp. 93-129, , https://doi.org/10.1007/978-1-4615-8103-1_3.

[25] S. Dew, T. Smy, M. Brett, Step coverage, uniformity and composition studies using integrated vapour transport and film-deposition models, Jpn. J. Appl. Phys. 33 (1994) 1140-1145, https://doi.org/10.1143/JJAP.33.1140.

[26] T. Smy, S.K. Dew, R.V. Joshi, Modeling 3D effects of substrate topography on step coverage and film morphology of thin metal films, Thin Solid Films 415 (2002) 32-45, https://doi.org/10.1016/S0040-6090(02)00502-3.

[27] G.A. Bird, Molecular Gas Dynamics and the Direct Simulation of Gas Flows, (1994)

[28] R. Tonneau, P. Moskovkin, A. Pflug, S. Lucas, TiOx deposited by magnetron sputtering: a joint modelling and experimental study, J. Phys. D Appl. Phys. 51 (2018), https://doi.org/10.1088/1361-6463/aabb72.

[29] A. Pflug, M. Siemers, B. Szyszka, Parallel DSMC Gasflow Simulation of an In-Line Coater for Reactive Sputtering, Springer, Berlin, Heidelberg, 2006, pp. 383-390, https://doi.org/10.1007/11846802 53.

[30] P.K. Petrov, V.A. Volpyas, R.A. Chakalov, Three-dimensional Monte Carlo simulation of sputtered atom transport in the process of ion-plasma sputter deposition of multicomponent thin films, Vacuum 52 (1999) 427-434, https://doi.org/10.1016/ S0042-207X(98)00326-1.

[31] K. Macek, P. Macek, U. Helmersson, Monte Carlo simulations of the transport of sputtered particles, ASDAM 1998 Conf. Proc. 2nd Int. Conf. Adv. Semicond. Devices Microsystems, 1998, https://doi.org/10.1109/ASDAM.1998.730167.

[32] J. Hereć, J. Sielanko, Z. Wroñski, Monte Carlo simulation of the concentration distribution of sputtered cathode material in glow discharge plasma, Vacuum 63 (2001) 507-512, https://doi.org/10.1016/S0042-207X(01)00230-5.

[33] S.N. Sambandam, S. Bhansali, V.R. Bhethanabotla, D.K. Sood, Studies on sputtering process of multicomponent Zr-Ti-Cu-Ni-Be alloy thin films, Vacuum 80 (2006) 406-414, https://doi.org/10.1016/j.vacuum.2005.07.037.

[34] G.S. Grest, B. Dünweg, K. Kremer, Vectorized link cell Fortran code for molecular dynamics simulations for a large number of particles, Comput. Phys. Commun. 55 (1989) 269-285, https://doi.org/10.1016/0010-4655(89)90125-2.

[35] A.M. Myers, J.R. Doyle, D.N. Ruzic, Monte Carlo simulations of sputter atom transport in low-pressure sputtering: the effects of interaction potential, sputter distribution, and system geometry, J. Appl. Phys. 72 (1992) 3064-3071, https:// doi.org/10.1063/1.351464.

[36] J. Geiser, S. Blankenburg, Monte Carlo Simulations of elastic scattering with applications to DC and high power pulsed magnetron sputtering for Ti 3SiC 2, Commun. Comput. Phys. 11 (2012) 1618-1642, https://doi.org/10.4208/cicp. 210211.270511a.

[37] A. Smith, Monte Carlo Simulation for silicon on silane HWCVD for thin film solar cells, Macalester J. Phasics Astron. 1 (2013).

[38] J.J. Jiménez, R.J. Segura, F.R. Feito, A robust segment/triangle intersection algorithm for interference tests. Efficiency study, Comput. Geom. Theory Appl. 43 (2010) 474-492, https://doi.org/10.1016/j.comgeo.2009.10.001.

[39] D. Depla, W.P. Leroy, Magnetron sputter deposition as visualized by Monte Carlo modeling, Thin Solid Films 520 (2012) 6337-6354, https://doi.org/10.1016/j.tsf. 2012.06.032.

[40] M.A. Hassan, M.B. Elfiky, R. Mahmoodian, Monte Carlo simulation model for magnetron sputtering deposition, Adv. Mater. Res. 1105 (2015) 69-73, https://doi org/10.4028/www.scientific.net/AMR.1105.69.

[41] W. Mattson, B.M. Rice, Near-neighbor calculations using a modified cell-linked list method, Comput. Phys. Commun. 119 (1999) 135-148, https://doi.org/10.1016/ S0010-4655(98)00203-3.

[42] T. Smy, M.J. Brett, Modelling and characterization 226 (1993) 196-201.

[43] A. Siad, A. Besnard, C. Nouveau, P. Jacquet, Critical angles in DC magnetron glad thin films, Vacuum 131 (2016) 305-311, https://doi.org/10.1016/j.vacuum.2016. 07.012. 